\title{
Effects of Platelet Rich Plasma in Combination with Hyaluronic Acid in the Treatment of Primary Knee Osteoarthritis
}

Md. Saydur Rahman', Syed Ariful Islam², Md. Jobayer Hossain³, Muhammad Shamsul Arefin', Md. Imrul Islam 5 , Sharmin Ara Begum ${ }^{6}$, Shofina Sultana Shompa ${ }^{7}$, AKM Akhtaruzzaman $^{8}$

\begin{abstract}
${ }^{1}$ Assistant Professor, Department of Anaesthesiology, Green Life Medical College and Hospital, Dhaka, Bangladesh; ${ }^{2}$ Emergency Medical Officer, 250 Bedded Brahmanbaria District Hospital, Brahmanbaria, Bangladesh; ${ }^{3}$ Associate Consultant, Department of Anaesthesiolgy, Asgar

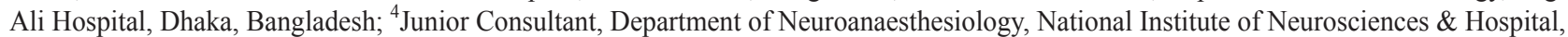

Dhaka, Bangladesh; ${ }^{5}$ Medical Officer, Department of Anaesthesia, Rajshahi Medical College Hospital, Rajshahi, Bangladesh; ${ }^{6}$ Post Graduate Student (MD Resident), Department of Anaesthesiology, Bangabandhu Sheikh Mujib Medical University, Dhaka, Bangladesh; ${ }^{7}$ Medical Officer, Department of Anaesthesiology, Bangabandhu Sheikh Mujib Medical University, Dhaka, Bangladesh; ${ }^{8}$ Chairman, Department of Anaesthesia, Analgesia \& Intensive Care Medicine, Bangabandhu Sheikh Mujib Medical University, Dhaka, Bangladesh
\end{abstract}

[Received: 10 April 2019 Accepted: 12 May 2019; Published: 1 July 2019]

\begin{abstract}
Background: Platelet Rich Plasma (PRP) is increasingly applied in clinical practice to treat knee degenerative pathology. Both PRP and hyaluronic acid (HA) have been extensively used to improve lubrication, modulate inflammation and modify the catabolic micro-environment of the joint. Objective: This study was aimed to evaluate the potential synergistic effects of PRP in combination with hyaluronic acid in primary knee osteoarthritis. Methodology: This randomized control trial was carried out on adult patients with primary knee osteoarthritis who attended the Pain Clinic of Bangabandhu Sheikh Mujib Medical University, Dhaka, Bangladesh, during the period of September 2015 to August 2017. The patients were randomly assigned to one of the two groups designated as group P (control group) who were only treated with PRP and group PH (experimental group) who were treated with the combination of PRP and hyaluronic acid. After providing the allocated treatment, all patients were undergone follow-up examination at 1st week, 1 st month and 3rd month for pain improvement by VAS scale and for functional improvement by International Knee Documentation Committee (IKDC) score. Results: A total number of 34 patients were recruited for this study. Visual analogue score 1st month $(5.85 \pm 0.83)$ and 3rd month $(3.01 \pm 0.12)$ follow up showed statistically significant difference among two group $(\mathrm{p}=0.001)$. Inter group comparison showed that,

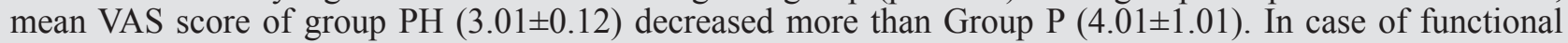
improvement, group PH also showed statistically better improvement during 1 st month $(45.76 \pm 3.25)$ and 3rd month (62.06 \pm 5.37$)$ follow up than Group P. Conclusion: In conclusion articular platelet rich plasma in combination with hyaluronic acid provide better pain relief and improvement of functional status than PRP therapy alone in primary knee osteoarthritis. [Journal of National Institute of Neurosciences Bangladesh, 2019;5(2): 137-142]
\end{abstract}

Keywords: Platelet rich plasma; hyaluronic acid; knee osteoarthritis; pain

Correspondence: Dr. Md. Saydur Rahman, Assistant Professor, Department of Anaesthesiology, Green Life Medical College and Hospital, Dhaka, Bangladesh; Cell no.: +8801715175218; Email: drsaydurrahman86@gmail.com

Conflict of interest: There is no conflict of interest relevant to this paper to disclose.

Funding agency: This research project was partially funded by Bangabandhu Sheikh Mujib Medical University, Dhaka.

Contribution to authors: Rahman MS, Islam SA, Hossain MJ have contributed from the protocol preparation, data collection up to report writing. Manuscript writing was performed by Arefin MS, Islam MI, Begum SA, Shompa SS, Naheen T, Das S, Arif NUM. Statistical analysis was performed by Akhtaruzzaman AKM. Rahman MS has involved in revision of manuscript.

How to cite this article: Rahman MS, Islam SA, Hossain MJ, Arefin MS, Islam MI, Begum SA, Shompa SS, Akhtaruzzaman AKM. Effects of Platelet Rich Plasma in Combination with Hyaluronic Acid in the Treatment of Primary Knee Osteoarthritis. J Natl Inst Neurosci Bangladesh, 2019;5(2): 137-142

Copyright: (C2019. Rahman et al. Published by Journal of National Institute of Neurosciences Bangladesh. This article is published under the Creative Commons CC BY-NC License (https://creativecommons.org/licenses/by-nc/4.0/). This license permits use, distribution and reproduction in any medium, provided the original work is properly cited, and is not used for commercial purposes.

\section{Introduction}

Osteoarthritis is a disorder involving joints characterized by cell stress and extracellular matrix degradation initiated by micro and macro-injury that activates maladaptive repair responses including pro-inflammatory pathways of innate immunity ${ }^{1}$.This condition impairs functional capacity and decrease quality of life (QOL) in patient's by producing pain, stiffness and limitation in range of motion of the joint ${ }^{2}$.

Hyaline cartilage enables almost frictionless joint 
movement and protects the underlying bone from excessive load and trauma ${ }^{3-4}$. In osteoarthritis, a combination of mechanical, cellular, and biochemical processes, resulting in abnormal repair response of cartilage that ultimately leads to cartilage injury ${ }^{5-7}$. Once cartilage is injured, it gradually degenerates, and leads to osteoarthritis.

Various conservative treatment modalities are recommended by various clinical guidelines for treatment of knee osteoarthritis. The nonpharmacological modalities are patient education and self-management, exercises, weight reduction, transcutaneous electrical nerve stimulation therapy. Pharmacologic therapies are paracetamol, non-steroidal anti-inflammatory drugs (NSAIDs), opioids, and slow-acting drugs (glucosamine and chondroitin sulfate). If orally administered drugs are ineffective, intra-articular (IA) injection (corticosteroids, visco supplements, blood-derived products) is the last non-operative modality that can be preferred ${ }^{8}$.

Although some of these treatment have short and mid-term effect on improving patients function and decreasing pain but none of these options has shown to delay the progression of the disease or reverse joint damage ${ }^{9}$. Beside these, various side effect has limited the use of NSAID and corticosteroid injection ${ }^{10,11}$. Considering these factors, current research is investigating new methods to stimulate repair or replace damaged cartilage to provide long term benefit. Platelet Rich Plasma (PRP) is a simple, low- cost and minimally invasive method containing a natural concentrate of autologous growth factors from the blood to provide the biological environment of the growing cells with a combination of all factors that are needed to initiate the healing process ${ }^{12}$

Platelets have a high concentration of growth factors and cytokines within their alpha and dense granules. Alpha granules of the platelet contains several vital growth factors like platelet-derived growth factor (PDGF), transforming growth factor-beta (TGF-b), insulin-like growth factor-1 (IGF-1), vascular endothelial growth factor (VEGF), and epidermal growth factor (EGF) among others. Beside these the dense granules of the platelet contain neuromodulators and inflammatory mediators such as histamine and serotonin. All the aforementioned growth factors and cytokines may impact soft tissue healing and bone regeneration ${ }^{13}$. Both PRP and hyaluronic acid (HA) have been extensively used for OA treatment to improve lubrication, modulate inflammation and modify the catabolic microenvironment not only for reducing clinical symptoms, but also to interfere with OA progression.

Hyaluronic acid facilitates the molecular pool released from PRP to reach the target cells by creating a pericellular bioactive scaffold around the cells. This pericellular matrix facilitate molecular diffusion and also adequate presentation of cytokines to their trans-membrane receptors located in the cytoplasmic membrane of the target competent cell. Another advantage of HA and PRP combination is facilitation of cells division and migration as this is a common hallmark of matrices that are rich in $\mathrm{HA}^{9}$.

Sundman et $\mathrm{al}^{14}$ showed in his basic clinical study that, separately hyaluronic acid and platelet rich plasma are beneficial for joint cells although they function through different mechanisms andb hypothesized that their combination may be synergistic. A synergistic anabolic actions of hyaluronic acid and platelet rich plasma combination has been also demonstrated in a 3D arthritic neo-cartilage and anterior cruciate ligament transection (ACLT-OA) model of animal'.

The purpose of the present study is to assess the potential synergistic effects of platelet rich plasma in combination with hyaluronic acid in primary knee osteoarthritis and to evaluate the effectiveness PRP as mono therapy and compare their effectiveness on chronic knee pain.

\section{Methodology}

This randomized control trial was conducted from September 2015 to August 2017 at Pain Clinic, Bangabandhu Sheikh Mujib Medical University, Bangladesh. It was a parallel designed, single blinded and single center study. After institutional ethical Board approval patients aged between 40 to 60 years, with symptomatic knee osteoarthritis for at least 4 months, not responding to conservative treatment with radiological evidences of K-L grade I and II were enrolled into the study. Patients refusal along with blood diseases, hepatitis B or C, HIV-positive status, infection and septicemia, local infection, severe cardiovascular diseases and immunosuppression, advanced and tri-compartmental osteoarthritis, rheumatoid or polyarticular arthritis, significant joint swelling or clinical signs of acute inflammation, anatomical deformity (Varus-valgus mal-alignment above $5^{\circ}$, patella-femoral maltracking or untreated instability), and total or subtotal meniscectomy (more than $2 / 3$ excised), pretreatment blood platelets value $25 \%$ below the reference value, treatment with corticosteroids less than 3 months or medication less than 7 days that could interfere with platelet aggregation and patients on therapy with 
anticoagulants-antiaggregants were excluded from the study. The patients were randomly assigned to one of the two groups where group $\mathrm{P}$ (control group) treated with only PRP therapy and group PH (experimental group) treated with combination of PRP and hyaluronic acid (correction made as per advice). They were advised to take prophylactic oral antibiotic tab. Cefixime 200 $\mathrm{mg}$ before starting of the procedure and to continue twice daily for 5 days. All procedures were performed in the operating room. After extraction of $18 \mathrm{~mL}$ of peripheral blood using a syringe pre-treated with $2 \mathrm{ml}$ of anticoagulant Citrate Dextrose Solution Formula (ACDA), sample was transferred to a sterile PRP kit (Dr. PRP USA LLC, Texus, USA). Then the kit was placed for two stage centrifugation according to recommendations of the manufacturer. We used REMI PRP (Remi group, Maharashtra, India.) device to obtain platelet rich plasma (PRP) form whole blood. After sterile dressing and draping intra articular injection was given to the patient in the supine position, with the knee partially flexed, using the lateral approach by 22 gauge needle. For Group P patient, freshly prepared $3 \mathrm{ml}$ autologous PRP were given intra-articularly and for group PH patient, immediately after introducing needle $3 \mathrm{ml}$ freshly prepared autologous PRP was given first, then $2 \mathrm{ml}$ of prefilled sodium hyaluronate was introduced through the same needle. After injection, all patients were instructed for early continuous passive movement to facilitate its diffusion and to apply ice at the injected site for 20 minutes at every 2-3 hours interval for 24 hour and to avoid NSAIDs and vigorous activities for at least 48 hour. All patients were undergone follow-up examination at 1st week, 1st month and 3rd month after the procedure by personal visit at Pain Clinic. Visual analog scale was used to assess the severity of pain and International Knee Documentation Committee (IKDC) score was used to assess the functional improvement. All patients were advised to follow Activities of Daily Living Instructions (ADLs) for knee osteoarthritis. Statistical analyses were carried out by using the Statistical Package for Social Sciences version 20.0 for Windows (SPSS Inc., Chicago, Illinois, USA). A descriptive analysis was performed for all data. The mean values were calculated for continuous variables. The qualitative observations were presented as frequencies and percentages. Unpaired t-test was used to compare continuous variables between control and experimental group. Chi-square test was used to compare categorical data like clinical signs and symptoms. A "p" value $<0.05$ was considered as significant.

\section{Results}

Two groups matched statistically comprising age, weight, sex, OA grade, BMI having no difference between them (Table 1).

Table 1: Characteristics of studied subject in two groups

\begin{tabular}{lccc}
\hline Character(s) & Group P & Group PH & P value \\
\hline $\begin{array}{l}\text { Age (in years) } \\
\text { Mean } \pm \text { SD }\end{array}$ & $48.53 \pm 4.93$ & $48.82 \pm 2.56$ & $0.830 \mathrm{~ns}$ \\
$\begin{array}{lcc}\text { Sex } \\
\quad \text { Male }\end{array}$ & $12(70.6)$ & $12(70.6)$ & $1.000 \mathrm{~ns}$ \\
$\quad$ Female & $5(29.4)$ & $5(29.4)$ & \\
\multicolumn{2}{l}{ Radiological OA Grade } & & \\
$\quad$ Grade I & $6(35.3)$ & $6(35.3)$ & $1.000 \mathrm{~ns}$ \\
$\quad$ Grade II & $11(64.7)$ & $11(64.7)$ & \\
Dominant knee involvement & & \\
$\quad$ Right side & $5(29.4)$ & $5(29.4)$ & $1.000 \mathrm{~ns}$ \\
$\quad$ Lift side & $12(70.6)$ & $12(70.6)$ & \\
Weight (In kg) & $58.12 \pm 9.39$ & $56.41 \pm 5.1$ & $0.066 \mathrm{~ns}$ \\
Range (min,mix) & 52,72 & 49,61 & \\
BMI (kg/m2) & $23.06 \pm 2.36$ & $22.72 \pm 1.02$ & $0.589 \mathrm{~ns}$ \\
\hline
\end{tabular}

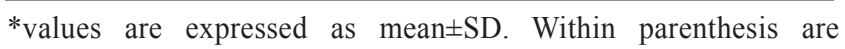
percentages over column total. ns= Not significant; $p$ value reached from unpaired $\mathrm{t}$-test and chi square test.

Table II shows VAS score at pretreatment and different follow up period. Mean VAS score at pretreatment were not statistically significant among two groups. Early follow up at 1st week VAS score was not significantly different but at later follow up during 1st month and 3rd month VAS score decreased more in group $\mathrm{PH}$ than group $\mathrm{P}$, which was statistically significant.

Table 2: VAS score at different follow up period of two studied groups

\begin{tabular}{lccc}
\hline VAS Score & Group P & Group PH & P value \\
\hline At pretreatment & $7.21 \pm 0.83$ & $7.65 \pm 0.51$ & $0.718 \mathrm{~ns}$ \\
At 1st week & $6.59 \pm 1.28$ & $5.85 \pm 0.83$ & $0.054 \mathrm{~ns}$ \\
At 1st month & $4.94 \pm 0.83$ & $3.35 \pm 0.83$ & $0.001 \mathrm{~s}$ \\
At 3rd month & $4.01 \pm 0.10$ & $3.01 \pm 0.12$ & $0.001 \mathrm{~s}$ \\
\hline
\end{tabular}

Values are expressed as Mean $\pm \mathrm{SD}, \mathrm{s}=$ significant, $\mathrm{ns}=$ non-significant, $\mathrm{p}$ value reached from student t-test.

Figure 1 shows functional outcome measured by International knee documentation committee (IKDC) score at pretreatment and different follow up period to assess the functional outcome in line diagram.

Mean IKDC score at pretreatment were not statistically significant among two groups. But at 1st week, 1st month and 3rd month follow up period, mean IKDC 
score was increased more in group PH than group P.At 1st week, 1st month and 3rd month outcome were statistically significant $(<0.05)$.

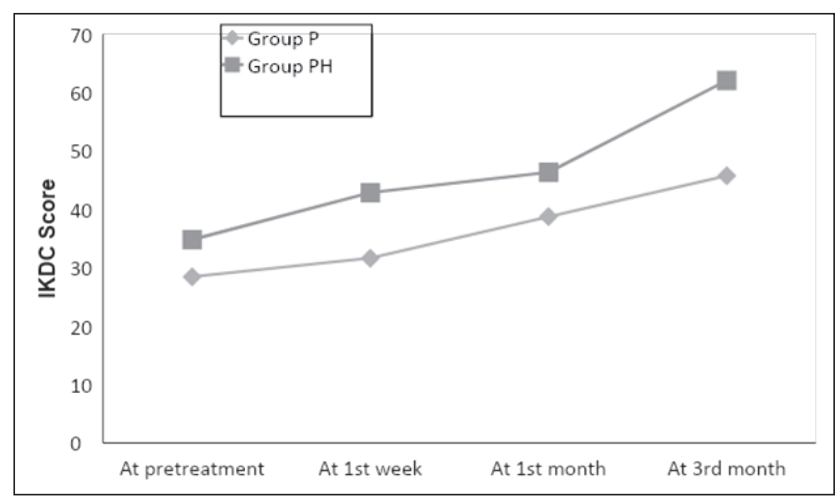

Figure 1: Line diagram shows mean IKDC score at different follow up period.

\section{Discussion}

Osteoarthritis being the most prevalent form of arthritis has become a major public health problem and causing pain and disability in one third of all affected patients ${ }^{15}$. This randomized clinical trial was carried out with the aims to assess the effectiveness of intra-articular platelet rich plasma (PRP) therapy alone versus combined PRP and hyaluronic acid (HA) therapy in relieving pain due to primary knee osteoarthritis, been evaluated by visual analog scale (VAS) for improvement of pain and functional outcome by international knee documentation committee (IKDC) score $^{16}$. This study also compare the outcome between two treatment groups.

All the demographic variables like age, sex, weight, BMI, OA K-L grading were almost alike between two groups and statistically non-significant. In this current study it was observed that mean VAS score at pretreatment were not statistically significant among two groups $(p=0.718)$. Early follow up at $1^{\text {st }}$ week VAS score was not significantly different but at later follow up during $1^{\text {st }}$ month and $3^{\text {rd }}$ month VAS score decreased more in group PB than group P, which was statistically significant.

In this current study functional outcomes were evaluated using the International Knee Documentation Committee (IKDC) scores. Mean IKDC score at 1st week, $1^{\text {st }}$ month and $3^{\text {rd }}$ month were statistically significant $(p<0.05)$ among two group. This study results showed that mean IKDC score was significantly $(p<0.05)$ increased in group PH than group P. Group $\mathrm{PH}$ had significantly more improvement $(\mathrm{p}=0.001)$ at all follow-up period.
Sanchez et $\mathrm{al}^{17}$ showed that PRP is better in pain, physical activity and overall WOMAC scores improvement upto 5 weeks compared to hyaluronic acid. Spakova et $\mathrm{al}^{18}$ showed statistically significant better results in the PRP group compared to HA at 3 and 6 month follow up periods in both WOMAC and numeric rating scale (NRS) scores. In another study Kon et $\mathrm{al}^{19}$ comparing PRP to hyaluronic acid concluded that autologous PRP injections provide more and longer efficacy than HA injections in reducing pain and symptoms and recovering articular function upto 6 month. In a placebo controlled randomized clinical trial, Patel et al20 treated 78 patients (156 knees) with bi-lateral osteoarthritis. Patients were divided randomly into 3 groups as Group A treated by a single PRP injection, group B by 2 PRP injections 3 weeks apart, and group $\mathrm{C}$ by single normal saline injection. Outcomes were evaluated by WOMAC and VAS score. It was verified that the groups treated with a single or double injection of PRP had an improvement in relation of placebo. Spaková et $\mathrm{al}^{18}$ and Lubowitz et $\mathrm{al}^{21}$ studies have suggested that the application of hyaluronic acid and PRP may have potentially positive effects on cartilage repair and slow down the progression of OA. Manunta et $\mathrm{a}^{22}$ assess the efficacy of associating the micro-fracture technique with platelet-rich plasma (PRP) injections in the treatment of chondral lesions to promote acceleration and optimization of the healing process. Microfracture is a technique in which the subchondral bone is penetrated to stimulate formation of a new articular surface. Their results also showed a better functional outcome (based on the IKDC score) in the patients treated with the combination of PRP and microfractures, even at 12 months.

However, there are many pre-clinical study that explains the success behind combination of hyaluronic acid and PRP therapy observed in this study. Studies show that HA provides appropriate matrix and supportive scaffold material for cartilage repair and enhances the mechanical properties of the cartilage ${ }^{23-25}$. Another study compared the effects of PRP or HA on inflammation, as measured by TNF- $\alpha$, IL- 6 and IL- 8 proteins; they found that, although both treatments decreased TNF- $\alpha$ production, IL- 6 was decreased only in HA cultures, but not in PRP treated cells, suggesting that both treatments influence inflammation through different mechanisms. The expression of catabolic enzymes like metalloproteinases was reduced in synoviocytes and chondrocytes treated with PRP but not in cells treated with HA. Thus, separately, HA and 
PRP are beneficial for joint cells although they function through different mechanisms. Thus they concluded that their combination may be synergistic ${ }^{14}$. Anitua et $\mathrm{al}^{26}$ evaluated the potential of pure PRP to induce tendon cells and synovial fibroblasts migration and examined whether the combination of PRP with HA improves their motility in vitro. Their study shows that PRP stimulated the migration of fibroblasts, as well as HA, but this effect was more prominent when HA was combined with PRP. Indeed, an increase of $33.5 \%$ in motility was observed in the case of HA and PRP treatment compared with HA. Therefore, this in vitro study definitely proves that PRP improves the biological properties of HA. The same has also been supported by Marmotti et $\mathrm{al}^{27}$ in his vitro study.

A synergistic anabolic actions of HA and PRP combination has been also demonstrated in a 3D arthritic neo-cartilage and anterior cruciate ligament transection (ACLT-OA) model of animal. Indeed, the combination of HA and PRP can synergistically promote cartilage regeneration and inhibit inflammation in the joint ${ }^{28}$.

Our study showed that this method of treatment is very safe as no complications such as infection or fever occurred among study subjects. Only minor adverse events were detected such as mild pain at injected area and skin bruises. Patel et $\mathrm{al}^{20}$ reported mild complications such as nausea and dizziness, which were of short duration but these complications were not reported in these patients.

Altogether, platelet rich plasma (PRP) has significant positive effect on patients with primary knee osteoarthritis and PRP in combination with hyaluronic acid provide better pain improvement and functional outcome than PRP alone.

\section{Conclusion}

The present study shows that intra articular platelet rich plasma in combination with hyaluronic acid provide better pain relief and improvement of functional status than PRP therapy alone in primary knee osteoarthritis. So combination treatment could be recognized as a future regenerative osteoarthritis therapy.

\section{References}

1. Kraus VB, Blanco FJ, Englund M, Karsdal MA, Lohmander LS. Call for standardized definitions of osteoarthritis and risk stratification for clinical trials and clinical use. Osteoarthr Cartil. 2015;23(8):1233-1241.

2. Zhu Y, Yuan M, Meng HY, et al. Basic science and clinical application of platelet-rich plasma for cartilage defects and osteoarthritis : a review. Osteoarthr Cartil. 2013;21(11):1627-1637.
3. Colledge NR, Walker BR, Ralston S, Davidson S. Davidson's Principles and Practice of Medicine. 22nd ed. /. Edinburgh ;;New York: Churchill Livingstone/Elsevier; 2014. http://www.worldcat.org/title/davidsons-principles-and-practice-o f-medicine/oclc/455157186. Accessed September 15, 2017.

4. Malfait A-M, Seymour AB, Gao F, et al. A role for PACE4 in osteoarthritis pain: evidence from human genetic association and null mutant phenotype. Ann Rheum Dis. 2012;71(6):1042-1048.

5. Walsh DA, McWilliams DF, Turley MJ, et al. Angiogenesis and nerve growth factor at the osteochondral junction in rheumatoid arthritis and osteoarthritis. Rheumatology. 2010;49(10): 1852-1861.

6. Hunter W. Of the Structure and Diseases of Articulating Cartilages, by William Hunter, Surgeon. Philos Trans R Soc London. 2011;42(462-471):514-521.

7. Hinton R, Moody RL, Davis AW TS. Osteoarthritis: diagnosis and therapeutic considerations. Am Fam Physician. 2002;65(5):841-848.http://www.ncbi.nlm.nih.gov/pubmed/ 22230308 .

8. Kon E, Filardo G, Drobnic M, et al. Non-surgical management of early knee osteoarthritis. Knee Surgery, Sport Traumatol Arthrosc. 2012;20(3):436-449.

9. Abate M, Andia I, Salini V. The Conservative Management of Osteoarthritis - Hyaluronic Acid, Platelet Rich Plasma or the Combination? In: Osteoarthritis - Progress in Basic Research and Treatment. InTech; 2015.

10. Adams RJ, Appleton SL, Gill TK, Taylor AW, Wilson DH, Hill CL. Cause for concern in the use of non-steroidal anti-inflammatory medications in the community -a population-based study. BMC Fam Pract. 2011;12(1):70.

11. Mader R, Lavi I, Luboshitzky R. Evaluation of the pituitary-adrenal axis function following single intraarticular injection of methylprednisolone. Arthritis Rheum. 2005;52(3): 924-928.

12. Tschon M, Fini M, Giardino R, Filardo G. Lights and shadows concerning platelet products for musculoskeletal regeneration. Front. 2011. https://www.bioscience.org/2011/v3e/af/224/2.htm. Accessed September 13, 2017.

13. Rodriguez-merchan EC. Intraarticular Injections of Platelet-rich Plasma ( PRP ). 2013;5(1):5-8.

14. Sundman EA, Cole BJ, Karas V, et al. The anti-inflammatory and matrix restorative mechanisms of platelet-rich plasma in osteoarthritis. Am J Sports Med. 2014;42(1):35-41.

15. Monteforte P, Rovetta G. Sonographic assessment of soft tissue alterations in osteoarthritis of the knee Sonographic assessment of soft tissue alterations in osteoarthritis of the knee. 1999;(December 2013).

16. Filardo G, Kon E, Roffi A, Di Matteo B, Merli ML, Marcacci M. Platelet-rich plasma: why intra-articular? A systematic review of preclinical studies and clinical evidence on PRP for joint degeneration. Knee Surgery, Sport Traumatol Arthrosc. 2015;23(9):2459-2474.

17. M S, E A, J A, Jj A, I A. - Intra-articular injection of an autologous preparation rich in growth factors for. Clin Exp Rheumatol. 2008;26:910-913.

18. Spaková T, Rosocha J, Lacko M, Harvanová D, Gharaibeh A. Treatment of Knee Joint Osteoarthritis with Autologous Platelet-Rich Plasma in Comparison with Hyaluronic Acid. Am J Phys Med Rehabil. 2012;91(5):411-417.

19. Kon E, Mandelbaum B, Buda R, et al. Platelet-rich plasma intra-articular injection versus hyaluronic acid viscosupplementation as treatments for cartilage pathology: From early degeneration to osteoarthritis. Arthrosc - J Arthrosc Relat Surg. 2011;27(11):1490-1501. 
20. Patel S, Dhillon MS, Aggarwal S, Marwaha N, Jain A. Treatment with platelet-rich plasma is more effective than placebo for knee osteoarthritis: A prospective, double-blind, randomized trial. Am J Sports Med. 2013;41(2):356-364.

21. Lubowitz JH, Provencher MT, Poehling GG. Cartilage treatment and biologics current research. Arthrosc - J Arthrosc Relat Surg. 2013;29(10):1597-1598.

22. Manunta F. J oints The treatment of chondral lesions of the knee with the. 2013;1(4):167-170.

23. Jfsd L, Weglein A, Sampson S, et al. By betwe Randomized controlled trial comparing hyaluronic acid, platelet-rich plasma and the combination of both in the treatment of mild and moderate osteoarthritis of the knee. 2016;12(2):69-78.

24. Levett PA, Hutmacher DW, Malda J, Klein TJ. Hyaluronic acid enhances the mechanical properties of tissue-engineered cartilage constructs. PLoS One. 2014;9(12).

25. Matsiko A, Levingstone TJ, O'Brien FJ, Gleeson JP. Addition of hyaluronic acid improves cellular infiltration and promotes early-stage chondrogenesis in a collagen-based scaffold for cartilage tissue engineering. J Mech Behav Biomed Mater. 2012;11:41-52.

26. Anitua E, Sanchez M, De la Fuente M, Zalduendo MM, Orive G. Plasma rich in growth factors (PRGF-Endoret) stimulates tendon and synovial fibroblasts migration and improves the biological properties of hyaluronic acid. Knee Surgery, Sport Traumatol Arthrosc. 2012;20(9):1657-1665.

27. Marmotti A, Rossi R, Castoldi F, Roveda E, Michielon G, Peretti GM. PRP and Articular Cartilage: A Clinical Update. 2015;2015.

28. Chen WH, Lo WC, Hsu WC, et al. Synergistic anabolic actions of hyaluronic acid and platelet-rich plasma on cartilage regeneration in osteoarthritis therapy. Biomaterials. 2014;35(36):9599-9607. 